\title{
Extended biorthogonal matrix polynomials
}

\author{
Ayman SheHata
}

\begin{abstract}
The pair of biorthogonal matrix polynomials for commutative matrices were first introduced by Varma and Taşdelen in [22]. The main aim of this paper is to extend the properties of the pair of biorthogonal matrix polynomials of Varma and Tasdelen and certain generating matrix functions, finite series, some matrix recurrence relations, several important properties of matrix differential recurrence relations, biorthogonality relations and matrix differential equation for the pair of biorthogonal matrix polynomials $J_{n}^{(A, B)}(x ; k)$ and $K_{n}^{(A, B)}(x ; k)$ are discussed. For the matrix polynomials $J_{n}^{(A, B)}(x ; k)$, various families of bilinear and bilateral generating matrix functions are constructed in the sequel.
\end{abstract}

\section{INTRODUCTION}

There has become an increased interest important in the extension of the notion of the theory of orthogonal matrix polynomials and many of their properties in the last two decades. In [1-8, 10, 14, 16-21], Chebyshev, Konhauser, Hermite and Jacobi matrix polynomials were studied and several properties of our results were given for these families of matrix polynomials for matrices in $\mathbb{C}^{N \times N}$. In [22], Varma and Taşdelen introduced and studied a pair of biorthogonal matrix polynomials $J_{n}^{(A, B)}(x ; k)$ and $K_{n}^{(A, B)}(x ; k)$ where all eigenvalues $\mu$ of the matrices $A$ and $B$ with commutative matrices satisfy the condition $\operatorname{Re}(\mu)>-1$.

Motivated by the work of Varma and Taşdelen [22], on biorthogonal matrix polynomials the present paper aims at an extension of the various properties of the pair of biorthogonal matrix polynomials $J_{n}^{(A, B)}(x ; k)$ and $K_{n}^{(A, B)}(x ; k)$ as have been already outlined in the abstract of this paper. This paper is constructed as follows. In section 2 , some matrix recurrence relations, several important properties of matrix differential recurrence relations, matrix differential equation and more generating matrix functions are given for these biorthogonal matrix polynomials $J_{n}^{(A, B)}(x ; k)$ given by Varma and Taşdelen. The relations between Konhauser and biorthogonal

2010 Mathematics Subject Classification. Primary: 33C45, 33C50; Secondary: 15A60.

Key words and phrases. Matrix functional calculus, Biorthogonal matrix polynomials, Jacobi matrix polynomials, Generating matrix functions, Differential operator. 
matrix polynomials in this study are also indicated, and the biorthogonality of the matrix polynomials $J_{n}^{(A, B)}(x ; k)$ of the first kind over the interval $(-1,1)$ with respect to a weight matrix function is shown. In section 3 , the biorthogonality of the biorthogonal matrix polynomials $K_{n}^{(A, B)}(x ; k)$ of the second kind over the interval $(-1,1)$ with respect to a weight matrix function $(1-x)^{A}(1+x)^{B}$ is given, several families of generating matrix functions and finite summation formula for these matrix polynomials $K_{n}^{(A, B)}(x ; k)$ of the second kind are shown. Various families of bilinear and bilateral generating matrix functions are derived for the matrix polynomials $J_{n}^{(A, B)}(x ; k)$ of the first kind. They obtained results are given as application of given in section 4 .

1.1. Preliminaries. For the sake of clarity in the subsection, we recall below definitions and some properties of matrix functional calculus, which will be used throughout this paper. All through this paper, its spectrum is denoted by $\sigma(A)$ where is the set of all eigenvalues of a matrix $A$ in $\mathbb{C}^{N \times N}$. The two-norm of a matrix $A$ is described by

$$
\|A\|=\sup _{x \neq 0} \frac{\|A x\|_{2}}{\|x\|_{2}}
$$

where $\|x\|_{2}=\left(x^{H} x\right)^{\frac{1}{2}}$ denotes the well-known Euclidean norm of a vector $x$ in $\mathbb{C}^{N}$. The identity and null matrix in $\mathbb{C}^{N \times N}$ will be denoted $I$ and $\mathbf{0}$, respectively.

Theorem 1.1. For functions $U(z)$ and $V(z)$ in an open set $\Omega$ are holomorphic functions of the complex plane. The matrices $P$ and $Q$ in $\mathbb{C}^{N \times N}$ are commutative with $\sigma(P) \subset \Omega$ and $\sigma(Q) \subset \Omega$, then from the matrix functional calculus [9], we have

$$
U(P) V(Q)=V(Q) U(P) \text { and } P Q=Q P .
$$

Lemma 1.1. If $A(k, n)$ is a matrix in $\mathbb{C}^{N \times N}$ for $k, n \in \mathbb{N}_{0}$, the following relations are satisfied (see, Defez and Jódar [7])

$$
\sum_{n=0}^{\infty} \sum_{k=0}^{\infty} A(k, n)=\sum_{n=0}^{\infty} \sum_{k=0}^{n} A(k, n-k)
$$

and

$$
\sum_{n=0}^{\infty} \sum_{k=0}^{n} A(k, n)=\sum_{n=0}^{\infty} \sum_{k=0}^{\infty} A(k, n+k) .
$$

Definition 1.1 ([11]). For a positive stable matrix $P$ in $\mathbb{C}^{N \times N}$, the Gamma matrix function $\Gamma(P)$ is described by

$$
\Gamma(P)=\int_{0}^{\infty} e^{-t} t^{P-I} d t ; \quad t^{P-I}=\exp ((P-I) \ln t) .
$$


Definition 1.2. For $A$ in $\mathbb{C}^{N \times N}$ such that $\sigma(A)$ does not contain 0 or a negative integer $\left(\sigma(A) \cap \mathbb{Z}^{-}=\emptyset\right.$ where $\emptyset$ is an empty set $)$, the matrix form of the Pochhammer symbol is given by

$$
\begin{aligned}
(A)_{n} & =A(A+I) \ldots(A+(n-1) I) \\
& =\Gamma(A+n I) \Gamma^{-1}(A) ; \quad n \in \mathbb{N} ; \quad(A)_{0}=I,
\end{aligned}
$$

where $\Gamma(A)$ is an invertible matrix.

Theorem $1.2([15])$. For $p$ and $q$ are finite positive integers and if $A_{i}$; $1 \leq i \leq p$ are matrices in $\mathbb{C}^{N \times N}$, and $B_{j} ; 1 \leq j \leq q$ are invertible matrices in $\mathbb{C}^{N \times N}$, and all matrices are commutative. The generalized hypergeometric matrix function satisfies the following matrix differential equation

$$
\left[\theta \prod_{j=1}^{q}\left(\theta I+B_{j}-I\right)-z \prod_{i=1}^{p}\left(\theta I+A_{i}\right)\right]{ }_{p} F_{q}=\boldsymbol{O} .
$$

Definition 1.3 ([11]). For positive stable matrices $P$ and $Q$ in $\mathbb{C}^{N \times N}$ with commutative matrices, then Beta matrix function $\mathbf{B}(P, Q)$ is given as

$$
\mathbf{B}(P, Q)=\int_{0}^{1} t^{P-I}(1-t)^{Q-I} d t .
$$

Lemma $1.2([8])$. Let $P$ and $Q$ be matrices in $\mathbb{C}^{N \times N}$ with commutative matrices such that

$$
\begin{aligned}
& \operatorname{Re}(z)>-1 \quad \text { for all } z \in \sigma(P), \\
& \operatorname{Re}(w)>-1, \quad \text { for all } w \in \sigma(Q) .
\end{aligned}
$$

Using (1.6), one can obtain

$$
\int_{-1}^{1}(1+x)^{P-I}(1-x)^{Q-I} d x=2^{P+Q-I} \boldsymbol{B}(P, Q) .
$$

Fact 1.1. For an arbitrary matrix $A \in \mathbb{C}^{N \times N}$,

$$
(1.9) D^{k}\left[t^{A+m I}\right]=(A+I)_{m}(A+I)_{m-k} t^{A+(m-k) I} ; k=0,1,2, \ldots, .
$$

Definition 1.4. The pair of the Konhauser matrix polynomials is given in [21] as follows:

$$
Z_{n}^{(A, \lambda)}(x ; k)=\Gamma(A+(k n+1) I) \sum_{m=0}^{n} \frac{(-1)^{m}(\lambda x)^{m k}}{(n-m) ! m !} \Gamma^{-1}(A+(k m+1) I)
$$

and

$$
Y_{n}^{(A, \lambda)}(x ; k)=\frac{1}{n !} \sum_{r=0}^{n} \sum_{s=0}^{r} \frac{(-1)^{s}(\lambda x)^{r}}{s !(r-s) !}\left(\frac{1}{k}((s+1) I+A)\right)_{n},
$$

where $\operatorname{Re}(\mu)>-1 \forall \mu \in \sigma(A)$ for $A \in \mathbb{C}^{N \times N}$ and $\lambda \in \mathbb{C}$ with $\operatorname{Re}(\lambda)>0$, $k \in \mathbb{N}=\mathbb{Z}^{+}$. 
Definition $1.5([8])$. Let $A$ and $B$ be commutative matrices in $\mathbb{C}^{N \times N}$ satisfying the condition

(1.12) $\operatorname{Re}(z)>-1, \forall z \in \sigma(A)$ and $\operatorname{Re}(w)>-1, \forall w \in \sigma(B)$.

For $n \geq 0$, the $n^{\text {th }}$ Jacobi matrix polynomials $P_{n}^{(A, B)}(x)$ is defined by the hypergeometric matrix function

$$
P_{n}^{(A, B)}(x)=\frac{(B+I)_{n}}{n !}{ }_{2} F_{1}\left(A+B+(n+1) I,-n I ; B+I ; \frac{1-x}{2}\right) .
$$

Definition 1.6. In [22], Varma and Taşdelen defined the pair of the biorthogonal matrix polynomials suggested by Jacobi matrix polynomials have the explicit representation as follows:

$$
\begin{aligned}
J_{n}^{(A, B)}(x ; k)= & \Gamma(A+(k n+1) I) \sum_{r=0}^{n} \frac{(-1)^{r}}{(n-r) ! r !}(A+B+(n+1) I)_{k r} \\
& \times \Gamma^{-1}(A+(k r+1) I)\left(\frac{1}{2}(1-x)\right)^{k r}
\end{aligned}
$$

and

$$
\begin{aligned}
K_{n}^{(A, B)}(x ; k)= & \frac{1}{n !} \Gamma(B+(n+1) I) \sum_{r=0}^{n} \sum_{s=0}^{r} \frac{(-1)^{s}}{s !(r-s) !}\left(\frac{1}{k}((s+1) I+A)\right)_{n} \\
& \times \Gamma^{-1}(B+(n-r+1) I)\left(\frac{1}{2}(1-x)\right)^{r}\left(\frac{1}{2}(x+1)\right)^{n-r}
\end{aligned}
$$

where $A$ and $B$ are commutative matrices in $\mathbb{C}^{N \times N}$ satisfying the conditions

$$
\begin{array}{ll}
\operatorname{Re}(\mu)>-1 & \text { for all eigenvalues } \mu \in \sigma(A) \text { and } \\
\operatorname{Re}(\nu)>-1 & \text { for all eigenvalues } \nu \in \sigma(B)
\end{array}
$$

and $k \in \mathbb{N}=\mathbb{Z}^{+}$.

Remark 1.1. If $k=1,(1.14)$ and (1.15) reduced to Jacobi matrix polynomials (1.13).

Theorem 1.3 ([22]). The matrix polynomials $J_{n}^{(A, B)}(x ; k)$ given by $(1.14)$ have the following generating matrix functions

$$
\begin{aligned}
& \sum_{n=0}^{\infty}(A+B+I)_{n}\left[(A+I)_{k n}\right]^{-1} J_{n}^{(A, B)}(x ; k) t^{n}=(1-t)^{-A-B-I} \\
& \times{ }_{k+1} F_{k}\left(\frac{A+B+I}{k+1}, \frac{A+B+2 I}{k+1}, \ldots, \frac{A+B+(k+1) I}{k+1} ;\right. \\
& \left.\frac{A+I}{k}, \frac{A+2 I}{k}, \ldots, \frac{A+k I}{k} ;\left(\frac{(k+1)(1-x)}{2 k}\right)^{k}\left(\frac{-(k+1) t}{(1-t)^{k+1}}\right)\right),
\end{aligned}
$$


for $|t|<1,|x|<1$ and $\left|\left(\frac{(k+1)(1-x)}{2 k}\right)^{k}\left(\frac{-(k+1) t}{(1-t)^{k+1}}\right)\right|<1$, and

$$
\begin{aligned}
& \sum_{n=0}^{\infty}\left[(A+I)_{k n}\right]^{-1} J_{n}^{(A, B-n I)}(x ; k) t^{n}=e^{t} \\
& \times{ }_{k} F_{k}\left(\frac{A+B+I}{k}, \frac{A+B+2 I}{k}, \ldots,\right. \\
& \left.\frac{A+B+k I}{k} ; \frac{A+I}{k}, \frac{A+2 I}{k}, \ldots, \frac{A+k I}{k} ;-t\left(\frac{(1-x)}{2}\right)^{k}\right) .
\end{aligned}
$$

Theorem $1.4([22])$. The matrix polynomials $J_{n}^{(A, B)}(x ; k)$ given by $(1.14)$ satisfy the following matrix recurrence relations

$$
\begin{aligned}
& (x-1) D J_{n}^{(A, B)}(x ; k)=n k J_{n}^{(A, B)}(x ; k) \\
& -k(A+(k n-k+1) I)_{k} J_{n-1}^{(A, B+I)}(x ; k)
\end{aligned}
$$

and

$(1.20)(x-1) D J_{n}^{(A, B)}(x ; k)=(A+k n I) J_{n}^{(A-I, B+I)}(x ; k)-A J_{n}^{(A, B)}(x ; k)$.

Fact 1.2. For any matrix $A$ in $\mathbb{C}^{N \times N}$ for $|z|<1$, we give the following relation (see Jódar and Cortés [12])

$$
(1-z)^{-A}={ }_{1} F_{0}(A ;-; z)=\sum_{n=0}^{\infty} \frac{1}{n !}(A)_{n} z^{n}
$$

2. SOME PROPERTIES OF BIORTHOGONAL

$$
\text { MATRIX POLYNOMIALS } J_{n}^{(A, B)}(x ; k)
$$

This section is devoted to introduce the finite summation, biorthogonality, some matrix recurrence relations, several matrix differential recurrence relations, matrix differential equation and more generating matrix functions for the biorthogonal matrix polynomials $J_{n}^{(A, B)}(x ; k)$ of the first kind.

Theorem 2.1. For matrices $A, B$ and $B+m I$ in $\mathbb{C}^{N \times N}$ satisfying the condition (1.16) and all matrices are commutative. Then biorthogonal matrix polynomials satisfy:

$$
\begin{aligned}
J_{n}^{(A, B)}(x ; k)= & \left(\frac{x-1}{y-1}\right)^{k n} \sum_{m=0}^{n} \frac{1}{m !}(A+I)_{k n}\left[(A+I)_{k(n-m)}\right]^{-1} \\
& \times\left[\left(\frac{y-1}{x-1}\right)^{k}-1\right]^{m} J_{n-m}^{(A, B+m I)}(y ; k) .
\end{aligned}
$$


Proof. If we put $y=\frac{1-x}{2}$ in (1.18) and replace $t$ by $t x^{k}$, we have

$$
\begin{aligned}
& e^{-t x^{k}} \sum_{n=0}^{\infty}\left[(A+I)_{k n}\right]^{-1} J_{n}^{(A, B-n I)}(1-2 y ; k) t^{n} x^{k n}={ }_{k} F_{k}\left(\frac{A+B+I}{k},\right. \\
& \left.\frac{A+B+2 I}{k}, \ldots, \frac{A+B+k I}{k} ; \frac{A+I}{k}, \frac{A+2 I}{k}, \ldots, \frac{A+k I}{k} ;-t x^{k} y^{k}\right)
\end{aligned}
$$

Interchange of $x$ and $y$ yields

$$
\begin{aligned}
& e^{-t y^{k}} \sum_{n=0}^{\infty}\left[(A+I)_{k n}\right]^{-1} J_{n}^{(A, B-n I)}(1-2 x ; k) t^{n} y^{k n}={ }_{k} F_{k}\left(\frac{A+B+I}{k},\right. \\
& \left.\frac{A+B+2 I}{k}, \ldots, \frac{A+B+k I}{k} ; \frac{A+I}{k}, \frac{A+2 I}{k}, \ldots, \frac{A+k I}{k} ;-t x^{k} y^{k}\right) .
\end{aligned}
$$

It is easy to see the right-hand sides of the above two expressions are the same. Thus, we get

$$
\begin{aligned}
& \sum_{n=0}^{\infty}\left[(A+I)_{k n}\right]^{-1} J_{n}^{(A, B-n I)}(1-2 y ; k) t^{n} x^{k n}= \\
& e^{t\left(x^{k}-y^{k}\right)} \sum_{n=0}^{\infty}\left[(A+I)_{k n}\right]^{-1} J_{n}^{(A, B-n I)}(1-2 x ; k) t^{n} y^{k n} .
\end{aligned}
$$

Equating the coefficients of $t^{n}$ on both sides gives the formula (2.1).

Theorem 2.2. The matrix polynomials $J_{n}^{(A, B)}(x ; k)$ satisfy the following biorthogonality condition

$$
\int_{-1}^{1} x^{s}(1-x)^{A}(1+x)^{B} J_{n}^{(A, B)}(x ; k) d x \begin{cases}=\boldsymbol{O}, & s=0,1,2, \ldots, n-1 \\ \neq \boldsymbol{O}, & s=n\end{cases}
$$

with respect to the weight matrix function $W(x, A, B)=(1-x)^{A}(1+x)^{B}$ over the interval $(-1,1)$ and the matrices $A$ and $B$ are commutative in $\mathbb{C}^{N \times N}$ satisfying the condition (1.16). 
Proof. If we replace $J_{n}^{(A, B)}(x ; k)$ by the form (1.14) in the left-hand side of (2.2), then carry out the permissible interchange of summation and integration to obtain

$$
\begin{aligned}
& \int_{-1}^{1} x^{s}(1-x)^{A}(1+x)^{B} J_{n}^{(A, B)}(x ; k) d x=\Gamma(A+(k n+1) I) \\
& \times \sum_{r=0}^{n} \frac{(-1)^{r}}{2^{k r}(n-r) ! r !}(A+B+(n+1) I)_{k r} \Gamma^{-1}(A+(k r+1) I) \\
& \times \int_{-1}^{1} x^{s}(1-x)^{A+k r I}(1+x)^{B} d x=\Gamma(A+(k n+1) I) \\
& \times \sum_{r=0}^{n} \frac{(-1)^{r}}{2^{k r}(n-r) ! r !}(A+B+(n+1) I)_{k r} \Gamma^{-1}(A+(k r+1) I) \\
& \times \sum_{m=0}^{s} \frac{(-1)^{m} s !}{m !(s-m) !} \int_{-1}^{1}(1-x)^{A+k r I}(1+x)^{B+m I} d x .
\end{aligned}
$$

Replacing $x$ by $(1+x)-1$, we get

$$
\begin{aligned}
& \int_{-1}^{1} x^{s}(1-x)^{A}(1+x)^{B} J_{n}^{(A, B)}(x ; k) d x=\Gamma(A+(k n+1) I) \\
& \times \sum_{r=0}^{n} \sum_{m=0}^{s} \frac{(-1)^{r+m}}{2^{k r}(n-r) !(s-m) ! m ! r !}(A+B+(n+1) I)_{k r} \\
& \times \Gamma^{-1}(A+(k r+1) I) \int_{-1}^{1}(1-x)^{A+k r I}(1+x)^{B+m I} d x \\
& =(-1)^{s} 2^{A+B+I} \Gamma(A+(k n+1) I) \Gamma(B+I) \Gamma^{-1}(A+B+(n+1) I) \\
& \times \sum_{r=0}^{n} \sum_{m=0}^{s} \frac{(-1)^{r+m}}{2^{k r}(n-r) !(s-m) ! m ! r !}(A+B+I)_{n+k r}(B+I)_{m} \\
& \times\left[(A+B+I)_{m+k r+1}\right]^{-1}=(-1)^{s} 2^{A+B+I} \Gamma(A+(k n+1) I) \Gamma(B+I) \\
& \times \Gamma^{-1}(A+B+(n+1) I) \sum_{r=0}^{n} \sum_{m=0}^{s} \frac{(-1)^{r+m}}{2^{k r}(n-r) !(s-m) ! m ! r !}(A+B+I)_{n+k r} \\
& \times(B+I)_{m} D^{n-m-1} x A+B+\left.(k n+n) I\right|_{x=1} \\
& =(-1)^{s} 2^{A+B+I} \Gamma(A+(k n+1) I) \Gamma(B+I) \Gamma^{-1}(A+B+(n+1) I) \\
& \times \sum_{r=0}^{n} \sum_{m=0}^{s} \frac{(-1)^{r+m}}{2^{k r}(n-r) !(s-m) ! m ! r !}(A+B+I)_{n+k r}(B+I)_{m} \\
& D^{n-m-1} x A+B+\left.n I\left(1-x^{k}\right)^{n}\right|_{x=1} .
\end{aligned}
$$


This matrix is a zero matrix or a null matrix for $0 \leq s \leq n-1$ and different from zero matrix or a null matrix for $s=n$.

By using integration by parts $n$ times yields

$$
\begin{aligned}
& \int_{-1}^{1} x^{n}(1-x)^{A}(1+x)^{B} J_{n}^{(A, B)}(x ; k) d x \\
& =2^{A+B+(n+1) I} \Gamma(A+(k n+1) I) \Gamma(B+(n+1) I) \\
& \times \Gamma^{-1}(A+B+(n+2) I)\left[\left(I+\frac{1}{k}(A+B+(n+1))\right)_{n}\right]^{-1} .
\end{aligned}
$$

Thus, the formula (2.3) means that

$$
\int_{-1}^{1} x^{s}(1-x)^{A}(1+x)^{B} J_{n}^{(A, B)}(x ; k) d x \begin{cases}=\mathbf{0}, & s=0,1,2, \ldots, n-1 ; \\ \neq \mathbf{0}, & s=n,\end{cases}
$$

which gives $(2.2)$.

Theorem 2.3. For the biorthogonal matrix polynomials $J_{n}^{(A, B)}(x ; k)$, we have the matrix differential recurrence relations

$$
\begin{aligned}
& D^{k}\left[(x-1)^{A+I} D J_{n}^{(A, B)}(x ; k)\right]=k(-1)^{k} 2^{-k}(A+B+(k n+1) I)_{k} \\
& \times(A+(k n-k+1) I)_{k}(1-x)^{A} J_{n-1}^{(A, B+(k+1) I)}(x ; k) ; n \geq 1
\end{aligned}
$$

and

$$
\begin{aligned}
& D^{k}\left[(x-1)^{A+I} D J_{n}^{(A, B)}(x ; k)\right]=k(-1)^{k} 2^{-k}(A+B+(n+1) I)_{k}(1-x)^{A} \\
& \times\left[(1-x) D J_{n}^{(A, B+(k+1) I)}(x ; k)+n k J_{n}^{(A, B+k I)}(x ; k)\right],
\end{aligned}
$$

where $A, B, B+k I$ and $B+(k+1) I$ are commutative matrices in $\mathbb{C}^{N \times N}$ satisfying the condition (1.16).

Proof. From (1.19), we get

$$
\begin{aligned}
& (x-1) D J_{n}^{(A, B)}(x ; k)=\frac{x-1}{n !} \Gamma(A+(k n+1) I) \Gamma^{-1}(A+B+(n+1) I) \\
& \times \sum_{r=0}^{n} \frac{(-1)^{r}}{2}\left(\begin{array}{l}
n \\
r
\end{array}\right) \Gamma(A+B+(k r+n+1) I) \Gamma^{-1}(A+(k r+1) I)\left(\frac{1}{2}(1-x)\right)^{k r-1} .
\end{aligned}
$$

This expression can be rewritten as

$$
\begin{aligned}
& (x-1) D J_{n}^{(A, B)}(x ; k)=\frac{k}{(n-1) !} \Gamma(A+(k n+1) I) \Gamma^{-1}(A+B+(n+1) I) \\
& \times \sum_{r=0}^{n}(-1)^{r}\left(\begin{array}{c}
n-1 \\
r-1
\end{array}\right) \Gamma(A+B+(k r+n+1) I) \Gamma^{-1}(A+(k r+1) I)\left(\frac{1}{2}(1-x)\right)^{k r} .
\end{aligned}
$$


Multiply both sides by $(1-x)^{A}$ and taking the $k$-th derivative, we have

$$
\begin{aligned}
& D^{k}\left[(x-1)^{A+I} D J_{n}^{(A, B)}(x ; k)\right]=\frac{k(-1)^{k}}{(n-1) !} \Gamma(A+(k n+1) I) \\
& \times \Gamma^{-1}(A+B+(n+1) I) \sum_{r=0}^{n} \frac{(-1)^{r}}{2^{k r}}\left(\begin{array}{c}
n-1 \\
r-1
\end{array}\right) \Gamma(A+B+(k r+n+1) I) \\
& \times \Gamma^{-1}(A+(k r-k+1) I)(1-x)^{A+(k r-k) I}=\frac{k(-1)^{k} 2^{-k}}{(n-1) !} \\
& \times \Gamma(A+(k n+1) I) \Gamma^{-1}(A+B+(n+1) I)(1-x)^{A} \sum_{r=0}^{n} \frac{(-1)^{r}}{2^{k r}}\left(\begin{array}{l}
n-1 \\
r-1
\end{array}\right) \\
& \times \Gamma(A+B+(k r+n+1) I) \Gamma^{-1}(A+(k r-k+1) I)\left(\frac{1}{2}(1-x)\right)^{(r-1) k} \\
& =\frac{k(-1)^{k} 2^{-k}}{(n-1) !}(A+B+(k n+1) I)_{n}(A+(k n-k+1) I)_{k}(1-x)^{A} \\
& \times \Gamma^{-1}(A+B+(k+n+1) I) \Gamma(A+(k n-k+1) I) \sum_{r=0}^{n-1} \frac{(-1)^{r}}{2^{k r}}\left(\begin{array}{l}
n-1 \\
r
\end{array}\right) \\
& \times \Gamma(A+B+(k r+n+1) I) \Gamma^{-1}(A+(k r+1) I)\left(\frac{1}{2}(1-x)\right)^{r k} .
\end{aligned}
$$

Hence yields the matrix differential recurrence relation in (2.4). The relation (2.5) can be obtained similarly from (2.4) and (1.19).

Theorem 2.4. For the matrices $A, A-I, A+k I, B$ and $B+I$ in $\mathbb{C}^{N \times N}$ with commutative matrices satisfying the condition (1.16). The biorthogonal matrix polynomials $J_{n}^{(A, B)}(x ; k)$ satisfy the interesting matrix recurrence relation

$$
\begin{aligned}
& k(1-x)^{k}(A+B+(n+1) I)_{k} J_{n-1}^{(A+k I, B+I)}(x ; k) \\
& =2^{k} A J_{n}^{(A, B)}(x ; k)-2^{k}(A+k n I) J_{n}^{(A-I, B+I)}(x ; k), n \geq 1 .
\end{aligned}
$$

Proof. If we denote the right-hand side of (1.18) by $F=F(x, t ; A, B ; k)$, one can easily verified that

$$
\begin{array}{r}
{ }_{k} F_{k}\left(\frac{A+B+(k+1) I}{k}, \frac{A+B+(k+2) I}{k}, \ldots, \frac{A+B+2 k I}{k}\right. \\
\left.; \frac{A+(k+1) I}{k}, \frac{A+(k+2) I}{k}, \ldots, \frac{A+2 k I}{k} ; z\right),
\end{array}
$$


which satisfies the following :

$$
\begin{aligned}
& k z \Gamma(A+B+(k+1) I) \Gamma^{-1}(A+(k+1) I){ }_{k} F_{k}\left(\frac{A+B+(k+1) I}{k},\right. \\
& \frac{A+B+(k+2) I}{k}, \ldots, \frac{A+B+2 k I}{k} ; \frac{A+(k+1) I}{k}, \frac{A+(k+2) I}{k}, \\
& \left.\ldots, \frac{A+2 k I}{k} ; z\right)=\Gamma(A+B) \Gamma^{-1}(A){ }_{k} F_{k}\left(\frac{A+B}{k}, \frac{A+B+I}{k},\right. \\
& \left.\ldots, \frac{A+B+(k-1) I}{k} ; \frac{A}{k}, \frac{A+I}{k}, \ldots, \frac{A+(k-1) I}{k} ; z\right) \\
& -A(A+B)^{-1} \Gamma(A+B+I) \Gamma^{-1}(A+I)_{k} F_{k}\left(\frac{A+B+I}{k}, \frac{A+B+2 I}{k},\right. \\
& \left.\ldots, \frac{A+B+k I}{k} ; \frac{A+I}{k}, \frac{A+2 I}{k}, \ldots, \frac{A+k I}{k} ; z\right) .
\end{aligned}
$$

Multiply both sides by $e^{t}$ after putting $z=-t\left(\frac{1-x}{2}\right)^{k}$, and taking account into the generating matrix function (1.18), we have the matrix recurrence relation (2.6) for the matrix polynomials $J_{n}^{(A, B)}(x ; k)$.

Theorem 2.5. Let $A, A-I, A+k I, B, B-I$ and $B+I$ be commutative matrices in $\mathbb{C}^{N \times N}$ satisfying the condition (1.16). The biorthogonal matrix polynomials satisfy the matrix differential recurrence relations

$$
\begin{aligned}
& (x-1)\left[(A+B+n I) D J_{n}^{(A, B)}(x ; k)+k(A+(k n-k+1) I)_{k}\right. \\
& \left.\times D J_{n-1}^{(A, B)}(x ; k)\right]=(A+B+n I)\left[n J_{n}^{(A, B)}(x ; k)\right. \\
& \left.-k(A+(k n-k+1) I)_{k} J_{n-1}^{(A, B)}(x ; k)\right] ; n \geq 1,
\end{aligned}
$$

$$
\begin{aligned}
& (x-1) D J_{n}^{(A, B)}(x ; k)-n J_{n}^{(A, B)}(x ; k) \\
& =-(A+I)_{k n}\left[(A+B+I)_{n}\right]^{-1} \sum_{m=0}^{n-1}(A+B+I)_{m}\left[(A+I)_{k m}\right]^{-1} \\
& \times\left[k(A+B+I) J_{m}^{(A, B)}(x ; k)+(k+1)(x-1) D J_{m}^{(A, B)}(x ; k)\right] ; n \geq 1
\end{aligned}
$$


and

$$
\begin{aligned}
& (x-1) D J_{n}^{(A, B)}(x ; k)-n J_{n}^{(A, B)}(x ; k)=(A+I)_{k n}\left[(A+B+I)_{n}\right]^{-1} \\
& \times \sum_{m=0}^{n-1}(-k)^{n-m}(A+B+I)_{m}\left[(A+I)_{k m}\right]^{-1} \\
& \times(A+B+(k m+m+1) I) J_{m}^{(A, B)}(x ; k) ; n \geq 1 .
\end{aligned}
$$

Proof. In order to prove the formulas (2.7), (2.8) and (2.9), differentiate partially the formula

$$
F(x, t ; A, B ; k)=\sum_{n=0}^{\infty}(A+B+I)_{n}\left[(A+I)_{k n}\right]^{-1} J_{n}^{(A, B)}(x ; k) t^{n},
$$

with respect to $x$ and $t$ where $F=F(x, t ; A, B ; k)$ is the right-hand side of eq. (1.17), we have the partial differential equation

$$
(1-x)(1+k t) \frac{\partial F}{\partial x}+k t(1-t) \frac{\partial F}{\partial t}=(A+B+I) k t F .
$$

This equation (2.10) can be rewritten in the following forms

$$
\begin{gathered}
(1-x) \frac{\partial F}{\partial x}+k t \frac{\partial F}{\partial t}=(A+B+I) k t F+k t^{2} \frac{\partial F}{\partial t}-(1-x) k t \frac{\partial F}{\partial x}, \\
(1-x) \frac{\partial F}{\partial x}+k t \frac{\partial F}{\partial t}=\frac{(A+B+I) k t}{1-t} F-\frac{(k-1)(1-x) t}{1-t} \frac{\partial F}{\partial x}
\end{gathered}
$$

and

$$
(1-x) \frac{\partial F}{\partial x}+k t \frac{\partial F}{\partial t}=\frac{(A+B+I) k t}{1+k t} F+\frac{k(k+1) t^{2}}{1+k t} \frac{\partial F}{\partial t} .
$$

If we substitute for $F$ in (2.11), we give

$$
\begin{aligned}
& \sum_{n=0}^{\infty}(A+B+I)_{n}\left[(A+I)_{k n}\right]^{-1}\left[(1-x) D J_{n}^{(A, B)}(x ; k)+n J_{n}^{(A, B)}(x ; k)\right] t^{n} \\
& =(A+B+I) k \sum_{n=0}^{\infty}(A+B+I)_{n}\left[(A+I)_{k n}\right]^{-1} J_{n}^{(A, B)}(x ; k) t^{n+1} \\
& +k \sum_{n=0}^{\infty}(A+B+I)_{n}\left[(A+I)_{k n}\right]^{-1} n J_{n}^{(A, B)}(x ; k) t^{n+1} \\
& -k(1-x) \sum_{n=0}^{\infty}(A+B+I)_{n}\left[(A+I)_{k n}\right]^{-1} D J_{n}^{(A, B)}(x ; k) t^{n+1} .
\end{aligned}
$$

Equating the coefficients of $t^{n}$ on both sides and simplifying, we obtain (2.7).

The matrix differential recurrence relations (2.8) and (2.9) can be obtained from (2.12) and (2.13) respectively, as the technique used in the proof of $(2.7)$. 
Remark 2.1. For $k=1$, formulas (2.7), (2.8) and (2.9) reduced to the wellknown matrix differential recurrence relations for Jacobi matrix polynomials in [8].

In fact $J_{n}^{(A, B)}(x ; k)$ has the generalized hypergeometric matrix function in the following form (see [22])

$$
\begin{aligned}
& J_{n}^{(A, B)}(x ; k)=\frac{1}{n !}(A+I)_{k n k+1} F_{k}\left(-n I, \frac{A+B+(n+1) I}{k},\right. \\
& \frac{A+B+(n+2) I}{k}, \ldots, \frac{A+B+(n+k+1) I}{k} ; \frac{A+I}{k}, \\
& \left.\frac{A+2 I}{k}, \ldots, \frac{A+k I}{k} ;\left(\frac{1-x}{2}\right)^{k}\right) .
\end{aligned}
$$

From (1.5) and (2.14), we obtain the matrix differential equation of order $k+1$

$$
\begin{array}{r}
{\left[\frac{x-1}{k} D \prod_{j=1}^{k}\left(\frac{x-1}{k} D I+\frac{A+j I}{k}-I\right)-\left(\frac{1-x}{2}\right)^{k}\left(\frac{x-1}{k} D-n\right)\right.} \\
\left.\times \prod_{i=1}^{k}\left(\frac{x-1}{k} D I+\frac{A+B+(n+i) I}{k}\right)\right] J_{n}^{(A, B)}(x ; k)=\mathbf{0},
\end{array}
$$

for $\left|\left(\frac{1-x}{2}\right)^{k}\right|<1$. Which can be written in the form

$$
\begin{aligned}
& {\left[2^{k}(x-1) D((x-1) D I+A+(1-k) I)_{k}-(1-x)^{k}((x-1) D-n k)\right.} \\
\times & \left.((x-1) D I+A+B+(n+1) I)_{k}\right] J_{n}^{(A, B)}(x ; k)=\mathbf{0},\left|\left(\frac{1-x}{2}\right)^{k}\right|<1 .
\end{aligned}
$$

Summary of this result is given in the following theorem.

Theorem 2.6. For the matrices $A$ and $B$ in $\mathbb{C}^{N \times N}$ with commutative matrices satisfying the condition (1.16), the biorthogonal matrix polynomials $J_{n}^{(A, B)}(x ; k)$ satisfy the matrix differential equation of order $k+1$ in (2.15).

Remark 2.2. For $k=1$, the matrix differential equation (2.15) reduces to the usual matrix differential equation satisfied by the Jacobi matrix polynomials [8]. 
Corollary 2.1. The connection between biorthogonal and Konhauser matrix polynomials is given as follows

$$
\begin{aligned}
& J_{n}^{(A, B)}(x ; k)=\Gamma^{-1}(A+B+(n+1) I) \\
& \times \int_{0}^{\infty} t^{A+B+n I} e^{-t} Z_{n}^{(A, 1)}\left(\frac{1-x}{2} t ; k\right) d t .
\end{aligned}
$$

Proof. Using the formula (1.3), the right-hand side of (2.17) can be written as

$$
\begin{aligned}
& \Gamma^{-1}(A+B+(n+1) I) \int_{0}^{\infty} t^{A+B+n I} e^{-t} Z_{n}^{(A, 1)}\left(\frac{1-x}{2} t ; k\right) d t \\
& =\Gamma^{-1}(A+B+(n+1) I) \Gamma(A+(k n+1) I) \sum_{r=0}^{n} \frac{(-1)^{r}}{(n-r) ! r !} \\
& \times \Gamma^{-1}(A+(k r+1) I)\left(\frac{1-x}{2}\right)^{k r} \int_{0}^{\infty} t^{A+B+(n+k r) I} e^{-t} d t .
\end{aligned}
$$

We evaluate integral by using Gamma matrix function and taking the necessary steps, we obtain (2.17).

3. Some PROPERTIES OF BIORTHOGONAL

$$
\text { MATRIX POLYNOMIALS } K_{n}^{(A, B)}(x ; k)
$$

Here, we establish some of the interesting properties of biorthogonal matrix polynomials $K_{n}^{(A, B)}(x ; k)$ of the second kind.

Theorem 3.1. For the matrices $A$ and $B$ in $\mathbb{C}^{N \times N}$ with commutative matrices satisfying (1.16). Then the matrix polynomials $K_{n}^{(A, B)}(x ; k)$ satisfy the biorthogonality with respect to weight matrix function $W(x, A, B)=$ $(1-x)^{A}(1+x)^{B}$ over the interval $(-1,1)$

$\int_{-1}^{1}(1-x)^{k m}(1-x)^{A}(1+x)^{B} K_{n}^{(A, B)}(x ; k) d x \begin{cases}=\boldsymbol{O}, & m=0,1,2, \ldots, n-1 ; \\ \neq \boldsymbol{O}, & m=n .\end{cases}$

Proof. Taking the $K_{n}^{(A, B)}(x ; k)$ given by (1.15) in the left-hand side of (3.1), we have

$$
\begin{aligned}
& \text { L.H.S. }=\int_{-1}^{1}(1-x)^{k m}(1-x)^{A}(1+x)^{B} K_{n}^{(A, B)}(x ; k) d x=\frac{1}{n !} \Gamma(B+(n+1) I) \\
& \times \sum_{r=0}^{n} \sum_{s=0}^{r} \frac{(-1)^{s}}{2^{n} s !(r-s) !} \Gamma^{-1}(B+(n-r+1) I)\left(\frac{1}{k}((s+1) I+A)\right)_{n} \\
& \times \int_{-1}^{1}(1-x)^{A+(r+k m) I}(1+x)^{B+(n-r) I} d x=\frac{2^{A+B+(k m+1) I}}{n !}
\end{aligned}
$$




$$
\begin{aligned}
& \times \Gamma(B+(n+1) I) \Gamma(A+(k m+1) I) \Gamma^{-1}(A+B+(k m+n+2) I) \\
& \times \sum_{r=0}^{n} \sum_{s=0}^{r} \frac{(-1)^{s}}{2^{n} s !(r-s) !}(A+(k m+1) I)_{r}\left(\frac{1}{k}(A+(s+1) I)\right)_{n} .
\end{aligned}
$$

Using the relation (see [22])

$$
(-m I)_{n}=\sum_{r=0}^{n} \sum_{s=0}^{r} \frac{(-1)^{s}}{s !(r-s) !}(A+(k m+1) I)_{r}\left(\frac{1}{k}(A+(s+1) I)\right)_{n},
$$

we get

$$
\begin{aligned}
L . H . S .= & \frac{(-m I)_{n}}{n !} 2^{A+B+(k m+1) I} \Gamma(A+(k m+1) I) \\
& \times \Gamma(B+(n+1) I) \Gamma^{-1}(A+B+(k m+n+2) I) .
\end{aligned}
$$

Since, this matrix is a zero matrix or a null matrix for $0 \leq m \leq n-1$ and different from not zero matrix or not null matrix for $m=n$. For $m=n$, we get the r.h.s of (3.1). This completes the proof of (3.1).

Now, we establish a proof of the generating matrix functions for biorthogonal matrix polynomials $K_{n}^{(A, B)}(x ; k)$ of the second kind which we state in the form of the following theorem.

Theorem 3.2. If $A$ and $B-n I$ are matrices in $\mathbb{C}^{N \times N}$ with commutative matrices satisfying the condition (1.16), then the matrix polynomials $K_{n}^{(A, B)}(x ; k)$ of the second kind satisfy the interesting formula:

$$
\begin{aligned}
\sum_{n=0}^{\infty} K_{n}^{(A, B-n I)}(x ; k) t^{n}= & \left(\frac{x+1}{2}\right)^{-B}\left(1-\frac{x+1}{2} t\right)^{-\frac{A+B+I}{k}} \\
& \times\left[\frac{x-1}{2}+\left(1-\frac{x+1}{2} t\right)^{\frac{1}{k}}\right]^{B} .
\end{aligned}
$$

Proof. From (1.21) and (1.18), we have

$$
\begin{aligned}
& \sum_{n=0}^{\infty} K_{n}^{(A, B-n I)}(x ; k)\left(\frac{2 t}{x+1}\right)^{n} \\
& =\sum_{n=0}^{\infty} \sum_{r=0}^{\infty} \sum_{s=0}^{r} \frac{(-B)_{r}(-r)_{s}}{n ! s ! r !}\left(\frac{A+(s+1) I}{k}\right)_{n}\left(\frac{x-1}{x+1}\right)^{r} t^{n} \\
& =\sum_{r=0}^{\infty} \sum_{s=0}^{r} \frac{(-B)_{r}(-r)_{s}}{s ! r !}\left(\frac{x-1}{x+1}\right)^{r} \sum_{n=0}^{\infty} \frac{1}{n !}\left(\frac{A+(s+1) I}{k}\right)_{n} t^{n}
\end{aligned}
$$




$$
\begin{aligned}
& =\sum_{r=0}^{\infty} \sum_{s=0}^{r} \frac{(-B)_{r}(-r)_{s}}{s ! r !}\left(\frac{x-1}{x+1}\right)^{r}(1-t)^{-\frac{A+(s+1) I}{k}} \\
& =(1-t)^{-\frac{A+I}{k}} \sum_{r=0}^{\infty} \frac{(-B)_{r}}{r !}\left(\frac{x-1}{x+1}\right)^{r} \sum_{s=0}^{r} \frac{(-1)^{s} r !}{s !(r-s) !}(1-t)^{-\frac{s}{k}} \\
& =(1-t)^{-\frac{A+I}{k}} \sum_{r=0}^{\infty} \frac{(-B)_{r}}{r !}\left(\frac{x-1}{x+1}\right)^{r}\left[1-(1-t)^{-\frac{1}{k}}\right]^{r} \\
& =\left(\frac{x+1}{2}\right)^{-B}(1-t)^{-\frac{A+B+I}{k}}\left[\frac{x-1}{2}+(1-t)^{\frac{1}{k}}\right]^{B} .
\end{aligned}
$$

Replacing $t$ by $\frac{x+1}{2} t$ in (3), we obtain (3.2).

Theorem 3.3. If $A, B$ and $B-n I$ are matrices in $\mathbb{C}^{N \times N}$ with commutative matrices satisfying the condition (1.19). Then the biorthogonal matrix polynomials $K_{n}^{(A, B)}(x ; k)$ of the second kind satisfy the interesting relation:

$$
\begin{aligned}
& K_{n}^{(A, B-n I)}(x ; k)=\frac{1}{n !}\left(\frac{x+1}{2}\right)^{n I-B} \\
& \times\left.\left\{\frac{\partial^{n}}{\partial t^{n}}(1-t)^{-\frac{A+B+I}{k}}\left[\frac{x-1}{2}+(1-t)^{\frac{1}{k}}\right]^{B}\right\}\right|_{t=0} .
\end{aligned}
$$

Proof. Using the generating matrix function (3), we have directly (3.4).

Theorem 3.4. If $A, B, C$ and $B+m I$ are matrices in $\mathbb{C}^{N \times N}$ with commutative matrices satisfying the condition (1.16), then the $K_{n}^{(A, B)}(x ; k)$ satisfy the finite summation formula:

$$
K_{n}^{(A, B)}(x ; k)=\sum_{m=0}^{n} \frac{1}{m !}\left(\frac{1}{k}(A-C)\right)_{m}\left(\frac{x+1}{2}\right)^{m} K_{n-m}^{(C, B+m I)}(x ; k) .
$$

Proof. In order to obtain a summation formula for the second kind biorthogonal matrix polynomials recall the generating matrix function (3.2), (1.1) and (1.21). Thus, we have

$$
\begin{aligned}
& \sum_{n=0}^{\infty} K_{n}^{(A, B-n I)}(x ; k) t^{n} \\
& =\left(\frac{x+1}{2}\right)^{-B}\left(1-\frac{x+1}{2} t\right)^{-\frac{A+B+I}{k}}\left[\frac{x-1}{2}+\left(1-\frac{x+1}{2} t\right)^{\frac{1}{k}}\right]^{B}
\end{aligned}
$$




$$
\begin{aligned}
& =\left(1-\frac{x+1}{2} t\right)^{\frac{C-A}{k}}\left(\frac{x+1}{2}\right)^{-B}\left(1-\frac{x+1}{2} t\right)^{-\frac{C+B+I}{k}} \cdot \\
& \quad\left[\frac{x-1}{2}+\left(1-\frac{x+1}{2} t\right)^{\frac{1}{k}}\right]^{B} \\
& =\left(1-\frac{x+1}{2} t\right)^{-\frac{A-C}{k}} \sum_{n=0}^{\infty} K_{n}^{(C, B-n I)}(x ; k) t^{n} \\
& =\sum_{n=0}^{\infty} \sum_{m=0}^{\infty} \frac{1}{m !}\left(\frac{1}{k}(A-C)\right)_{m}\left(\frac{x+1}{2}\right)^{m} K_{n}^{(C, B-n I)}(x ; k) t^{n+m} \\
& =\sum_{n=0}^{\infty} \sum_{m=0}^{n} \frac{1}{m !}\left(\frac{1}{k}(A-C)\right)_{m}\left(\frac{x+1}{2}\right)^{m} K_{n-m}^{(C, B-(n-m) I)}(x ; k) t^{n} .
\end{aligned}
$$

Comparing of the coefficients of $t^{n}$ and replacing $B$ by $B+n I$ in both sides gives (3.5).

Theorem 3.5. If $A-k n I$ and $B-n I$ are matrices in $\mathbb{C}^{N \times N}$ with commutative matrices which satisfy the condition in (1.16), then we have the relation:

$$
\begin{aligned}
\sum_{n=0}^{\infty} K_{n}^{(A-k n I, B-n I)}(x ; k) t^{n}= & \left(\frac{x+1}{2}\right)^{-B}\left(1+\frac{x+1}{2} t\right)^{\frac{A+(1-k) I}{k}} \\
& \times\left[1+\left(\frac{x+1}{2}-1\right)\left(1+\frac{x+1}{2} t\right)^{\frac{1}{k}}\right]^{B} .
\end{aligned}
$$

Proof. From (1.18) and (1.21), we have

$$
\begin{aligned}
& \sum_{n=0}^{\infty} K_{n}^{(A-k n I, B-n I)}(x ; k)\left(\frac{2 t}{x+1}\right)^{n} \\
& =\sum_{n=0}^{\infty} \sum_{r=0}^{\infty} \sum_{s=0}^{r} \frac{(-B)_{r}(-r)_{s}}{n ! s ! r !}\left(1-\frac{A+(s+1) I}{k}\right)_{n}\left(\frac{x-1}{x+1}\right)^{r}(-t)^{n} \\
& =\sum_{r=0}^{\infty} \sum_{s=0}^{r} \frac{(-B)_{r}(-r)_{s}}{s ! r !}\left(\frac{x-1}{x+1}\right)^{r} \sum_{n=0}^{\infty} \frac{1}{n !}\left(1-\frac{A+(s+1) I}{k}\right)_{n}(-t)^{n} \\
& =\sum_{r=0}^{\infty} \sum_{s=0}^{r} \frac{(-B)_{r}(-r)_{s}}{s ! r !}\left(\frac{x-1}{x+1}\right)^{r}(1+t)^{\frac{A+(s+1) I}{k}-1} \\
& =(1+t)^{\frac{A+(1-k) I}{k}} \sum_{r=0}^{\infty} \frac{(-B)_{r}}{r !}\left(\frac{x-1}{x+1}\right)^{r} \sum_{s=0}^{r} \frac{(-1)^{s} r !}{s !(r-s) !}(1+t)^{\frac{s}{k}}
\end{aligned}
$$




$$
\begin{aligned}
& =(1+t)^{\frac{A+(1-k) I}{k}} \sum_{r=0}^{\infty} \frac{(-B)_{r}}{r !}\left(\frac{x-1}{x+1}\right)^{r}\left[1-(1+t)^{\frac{1}{k}}\right]^{r} \\
& =\left(\frac{x+1}{2}\right)^{-B}(1+t)^{\frac{A+(1-k) I}{k}}\left[1+\frac{x-1}{2}(1+t)^{\frac{1}{k}}\right]^{B} .
\end{aligned}
$$

Thus, we have established the bilateral generating matrix function (3.6) for the biorthogonal matrix polynomials $K_{n}^{(A, B)}(x ; k)$ of the second kind.

\section{Bilinear AND BILATERAL GENERATING MATRIX FUnCTIONS}

In this section, we give theorems which establish several substantially more general families of bilinear and bilateral generating matrix functions for $J_{n}^{(A, B)}(x ; k)$ of the first kind defined by (1.17) as well as their applications. Using the same techniques considered in $[2,6,10,20]$, we can obtain the main results.

Theorem 4.1. Corresponding to a non-vanishing matrix function $\Omega_{\mu}\left(y_{1}, y_{2}\right.$, $\left.\ldots, y_{s}\right)$ of $s$ complex variables $y_{1}, y_{2}, \ldots, y_{s}, s \in \mathbb{N}$ and of complex order $\mu$, let us consider the following

$$
\Lambda_{\mu, \nu}\left(y_{1}, y_{2}, \ldots, y_{s} ; z\right)=\sum_{k=0}^{\infty} a_{k} \Omega_{\mu+\nu k}\left(y_{1}, y_{2}, \ldots, y_{s}\right) z^{k} ; a_{k} \neq 0, \mu, \nu \in \mathbb{C},
$$

where the coefficients $a_{k}$ are assumed to a non-vanishing in order for the matrix function on the left-hand side to be non-null. Suppose that

$$
\begin{aligned}
& \Psi_{n, m, \mu, \nu}\left(x ; y_{1}, y_{2}, \ldots, y_{s} ; \eta\right)=\sum_{r=0}^{\left[\frac{1}{m} n\right]} a_{r}(A+B+I)_{n-m r} \\
& \times\left[(A+I)_{k(n-m r)}\right]^{-1} J_{n-m r}^{(A, B)}(x ; k) \Omega_{\mu+\nu r}\left(y_{1}, y_{2}, \ldots, y_{s}\right) \eta^{r} ; n, m \in \mathbb{N},
\end{aligned}
$$

where $A$ and $B$ are matrices in $\mathbb{C}^{N \times N}$ satisfying the condition (1.16) and all matrices are commutative, and (as usual) $[\alpha]$ represents the greatest integer in $\alpha \in \mathbb{R}$. Then, for $|t|<1,|x|<1$ and $\left|\left(\frac{(k+1)(1-x)}{2 k}\right)^{k}\left(\frac{-(k+1) t}{(1-t)^{k+1}}\right)\right|<1$, we have

$$
\begin{aligned}
& \sum_{n=0}^{\infty} \Psi_{n, m, \mu, \nu}\left(x ; y_{1}, y_{2}, \ldots, y_{s} ; \frac{\eta}{t^{m}}\right) t^{n}=(1-t)^{-A-B-I} \\
& \times{ }_{k+1} F_{k}\left(\frac{A+B+I}{k+1}, \frac{A+B+2 I}{k+1}, \ldots, \frac{A+B+(k+1) I}{k+1} ; \frac{A+I}{k},\right. \\
& \left.\frac{A+2 I}{k}, \ldots, \frac{A+k I}{k} ;\left(\frac{(k+1)(1-x)}{2 k}\right)^{k}\left(\frac{-(k+1) t}{(1-t)^{k+1}}\right)\right) \\
& \times \Lambda_{\mu, \nu}\left(y_{1}, y_{2}, \ldots, y_{s} ; \eta\right) .
\end{aligned}
$$


Proof. For convenience, suppose that $S$ denote the first member of the assertion (4.3) of Theorem 4.1. Then, plugging the matrix polynomials $\Psi_{n, m, \mu, \nu}\left(x ; y_{1}, y_{2}, \ldots, y_{s} ; \frac{\eta}{t^{m}}\right)$.

In view of the definition (4.2) into the left-hand side of (4.3), we obtain

$$
\begin{aligned}
& \sum_{n=0}^{\infty} \Psi_{n, m, \mu, \nu}\left(x ; y_{1}, y_{2}, \ldots, y_{s} ; \frac{\eta}{t^{m}}\right) t^{n}=\sum_{n=0}^{\infty} \sum_{r=0}^{\left[\frac{1}{m} n\right]} a_{r}(A+B+I)_{n-m r} \\
& \times\left[(A+I)_{k(n-m r)}\right]^{-1} J_{n-m r}^{(A, B)}(x ; k) \Omega_{\mu+\nu r}\left(y_{1}, y_{2}, \ldots, y_{s}\right) \eta^{r} t^{n-m r} .
\end{aligned}
$$

Upon inverting the order of summation in (4.4) and replacing $n$ by $n=$ $n+m r$, we can write

$$
\begin{aligned}
& \sum_{n=0}^{\infty} \Psi_{n, m, \mu, \nu}\left(x ; y_{1}, y_{2}, \ldots, y_{s} ; \frac{\eta}{t^{m}}\right) t^{n} \\
& =\sum_{n=0}^{\infty} \sum_{r=0}^{\infty} a_{k}(A+B+I)_{n}\left[(A+I)_{k n}\right]^{-1} J_{n}^{(A, B)}(x ; k) \Omega_{\mu+\nu r}\left(y_{1}, y_{2}, \ldots, y_{s}\right) \eta^{k} t^{n} \\
& =\left[\sum_{n=0}^{\infty}(A+B+I)_{n}\left[(A+I)_{k n}\right]^{-1} J_{n}^{(A, B)}(x ; k) t^{n}\right]\left[\sum_{r=0}^{\infty} a_{r} \Omega_{\mu+\nu r}\left(y_{1}, y_{2}, \ldots, y_{s}\right) \eta^{r}\right] \\
& =(1-t)^{-A-B-I}{ }_{k+1} F_{k}\left(\frac{A+B+I}{k+1}, \frac{A+B+2 I}{k+1}, \ldots, \frac{A+B+(k+1) I}{k+1} ; \frac{A+I}{k},\right. \\
& \left.\frac{A+2 I}{k}, \ldots, \frac{A+k I}{k} ;\left(\frac{(k+1)(1-x)}{2 k}\right)^{k}\left(\frac{-(k+1) t}{(1-t)^{k+1}}\right)\right) \Lambda_{\mu, \nu}\left(y_{1}, y_{2}, \ldots, y_{s} ; \eta\right),
\end{aligned}
$$

which completes the proof of Theorem 4.1.

Expressing the multivariable matrix function $\Omega_{\mu+\nu k}\left(y_{1}, y_{2}, \ldots, y_{s}\right), k \in$ $\mathbb{N}_{0}$ and $s \in \mathbb{N}$ in terms of simpler matrix function of one variable and more variables, we give the applications of Theorem 4.1. In the following, we provide a class of bilateral generating matrix functions for the biorthogonal matrix polynomials of the first kind.

Corollary 4.1. Let

$$
\Lambda_{\mu, \nu}(y ; z)=\sum_{r=0}^{\infty} a_{r} J_{\mu+\nu r}^{(P, Q)}(y ; k) z^{r} ; a_{r} \neq 0, \mu, \nu \in \mathbb{N}_{0}
$$

and

$$
\begin{aligned}
\Psi_{n, m, \mu, \nu}(x ; y ; \eta)= & \sum_{r=0}^{\left[\frac{1}{m} n\right]} a_{r}(A+B+I)_{n-m r}\left[(A+I)_{k(n-m r)}\right]^{-1} \\
& \times J_{n-m r}^{(A, B)}(x ; k) J_{\mu+\nu r}^{(P, Q)}(y ; k) \eta^{r} ; n, m \in \mathbb{N}
\end{aligned}
$$


where $P$ and $Q$ are matrices in $\mathbb{C}^{N \times N}$ satisfying the condition (1.16) and all matrices are commutative, then we get

$$
\begin{aligned}
& \sum_{n=0}^{\infty} \Psi_{n, m, \mu, \nu}\left(x ; y ; \frac{\eta}{t^{m}}\right) t^{n}=(1-t)^{-A-B-I}{ }_{k+1} F_{k}\left(\frac{A+B+I}{k+1},\right. \\
& \frac{A+B+2 I}{k+1}, \ldots, \frac{A+B+(k+1) I}{k+1} ; \frac{A+I}{k}, \frac{A+2 I}{k}, \\
& \left.\ldots, \frac{A+k I}{k} ;\left(\frac{(k+1)(1-x)}{2 k}\right)^{k}\left(\frac{-(k+1) t}{(1-t)^{k+1}}\right)\right) \Lambda_{\mu, \nu}(y ; \eta)
\end{aligned}
$$

provided that each member of (4.5) exists.

Remark 4.1. Using the generating matrix function given by (1.17) for the biorthogonal matrix polynomials of the first kind, and taking $a_{r}=(P+Q+$ $I)_{r}\left[(P+I)_{k r}\right]^{-1}, \mu=0$ and $\nu=1$, we get

$$
\begin{aligned}
& \sum_{n=0}^{\infty} \sum_{r=0}^{\left[\frac{1}{m} n\right]}(A+B+I)_{n-m r}\left[(A+I)_{k(n-m r)}\right]^{-1}(P+Q+I)_{r}\left[(P+I)_{k r}\right]^{-1} \\
& \times J_{n-m r}^{(A, B)}(x ; k) J_{r}^{(P, Q)}(y ; k) \eta^{k} t^{n-m k}=(1-t)^{-A-B-I}(1-\eta)^{-P-Q-I} \\
& \times{ }_{k+1} F_{k}\left(\frac{A+B+I}{k+1}, \frac{A+B+2 I}{k+1}, \ldots, \frac{A+B+(k+1) I}{k+1} ; \frac{A+I}{k},\right. \\
& \left.\frac{A+2 I}{k}, \ldots, \frac{A+k I}{k} ;\left(\frac{(k+1)(1-x)}{2 k}\right)^{k}\left(\frac{-(k+1) t}{(1-t)^{k+1}}\right)\right) \\
& \times{ }_{k+1} F_{k}\left(\frac{P+Q+I}{k+1}, \frac{P+Q+2 I}{k+1}, \ldots, \frac{P+Q+(k+1) I}{k+1} ;\right. \\
& \left.\frac{P+I}{k}, \frac{P+2 I}{k}, \ldots, \frac{P+k I}{k} ;\left(\frac{(k+1)(1-y)}{2 k}\right)^{k}\left(\frac{-(k+1) \eta}{(1-\eta)^{k+1}}\right)\right)
\end{aligned}
$$

for $|t|<1,|x|<1,|\eta|<1,|y|<1,\left|\left(\frac{(k+1)(1-x)}{2 k}\right)^{k}\left(\frac{-(k+1) t}{(1-t)^{k+1}}\right)\right|<1$ and $\left|\left(\frac{(k+1)(1-y)}{2 k}\right)^{k}\left(\frac{-(k+1) \eta}{(1-\eta)^{k+1}}\right)\right|<1$.

Now, we give some special cases for several important generating matrix functions. Firstly, if we set $\Omega_{\mu+\nu r}(y)=Y_{n}^{(D, \lambda)}(y ; k)$ for $s=1$ in Theorem 4.1 , where the Konhauser matrix polynomials $Y_{n}^{(D, \lambda)}(x ; k)$ are defined by means of the generating matrix function in [15]:

$$
\sum_{n=0}^{\infty} Y_{n}^{(D, \lambda)}(x ; k) t^{n}=(1-t)^{-\frac{1}{k}(D+I)} \exp \left[\lambda x\left[1-(1-t)^{-\frac{1}{k}}\right]\right] ;|t|<1
$$


where $D$ is a matrix in $\mathbb{C}^{N \times N}$ satisfying the condition $\operatorname{Re}(\mu)>-1 \forall \mu \in$ $\sigma(D)$ and all matrices are commutative. Then we obtain a class of bilateral generating matrix functions for the biorthogonal matrix polynomials $J_{n}^{(A, B)}(x ; k)$ and the Konhauser matrix polynomials $Y_{n}^{(D, \lambda)}(x ; k)$ of the second kind.

Corollary 4.2. Let

$$
\Lambda_{\mu, \nu}(y ; z)=\sum_{r=0}^{\infty} a_{r} Y_{\mu+\nu r}^{(D, \lambda)}(y ; k) z^{r} ; a_{r} \neq 0, \mu, \nu \in \mathbb{N}_{0}
$$

and

$$
\begin{aligned}
\Psi_{n, m, \mu, \nu}(x ; y ; \eta)= & \sum_{r=0}^{\left[\frac{1}{m} n\right]} a_{r}(A+B+I)_{n-m r}\left[(A+I)_{k(n-m r)}\right]^{-1} \\
& \times J_{n-m r}^{(A, B)}(x ; k) Y_{\mu+\nu r}^{(D, \lambda)}(y ; k) \eta^{r} ; n, m \in \mathbb{N},
\end{aligned}
$$

where $D$ is a matrix in $\mathbb{C}^{N \times N}$ satisfying $\operatorname{Re}(\mu)>-1 \forall \mu \in \sigma(D)$ and all matrices are commutative. Then we have

$$
\begin{aligned}
& \sum_{n=0}^{\infty} \Psi_{n, m, \mu, \nu}\left(x ; y ; \frac{\eta}{t^{m}}\right) t^{n}=(1-t)^{-A-B-I}{ }_{k+1} F_{k}\left(\frac{A+B+I}{k+1},\right. \\
& \frac{A+B+2 I}{k+1}, \ldots, \frac{A+B+(k+1) I}{k+1} ; \frac{A+I}{k}, \frac{A+2 I}{k}, \ldots, \\
& \left.\frac{A+k I}{k} ;\left(\frac{(k+1)(1-x)}{2 k}\right)^{k}\left(\frac{-(k+1) t}{(1-t)^{k+1}}\right)\right) \Lambda_{\mu, \nu}(y ; \eta)
\end{aligned}
$$

provided that each member of (4.7) exists.

Remark 4.2. Using the generating matrix function (4.6) for the Konhauser matrix polynomials $Y_{r}^{(D, \lambda)}(y ; k)$ and taking $a_{r}=1, \mu=0$ and $\nu=1$, we have

$$
\begin{aligned}
& \sum_{n=0}^{\infty} \sum_{r=0}^{\left[\frac{1}{m} n\right]}(A+B+I)_{n-m r}\left[(A+I)_{k(n-m r)}\right]^{-1} J_{n-m r}^{(A, B)}(x ; k) Y_{r}^{(D, \lambda)}(y ; k) \eta^{r} t^{n-m r} \\
& =(1-t)^{-A-B-I}{ }_{k+1} F_{k}\left(\frac{A+B+I}{k+1}, \frac{A+B+2 I}{k+1}, \ldots, \frac{A+B+(k+1) I}{k+1}\right. \\
& \left.\frac{A+I}{k}, \frac{A+2 I}{k}, \ldots, \frac{A+k I}{k} ;\left(\frac{(k+1)(1-x)}{2 k}\right)^{k}\left(\frac{-(k+1) t}{(1-t)^{k+1}}\right)\right) \\
& \times(1-\eta)^{-\frac{1}{k}(D+I)} \exp \left[\lambda y\left[1-(1-\eta)^{-\frac{1}{k}}\right]\right] \\
& \text { for }|t|<1,|x|<1,|\eta|<1 \text { and }\left|\left(\frac{(k+1)(1-x)}{2 k}\right)^{k}\left(\frac{-(k+1) t}{(1-t)^{k+1}}\right)\right|<1 .
\end{aligned}
$$


Also setting $\Omega_{\mu+\nu r}(y)=Z_{n}^{(D, \lambda)}(y ; k)$ for $s=1$ in Theorem 4.1, where the Konhauser matrix polynomials of the second kind is defined by means of the generating matrix function

$$
\begin{aligned}
& \sum_{n=0}^{\infty}\left[(D+I)_{k n}\right]^{-1} Z_{n}^{(D, \lambda)}(x ; k) t^{n} \\
& =e^{t}{ }_{0} F_{k}\left(-; \frac{D+I}{k}, \frac{D+2 I}{k}, \ldots, \frac{D+k I}{k} ;-\frac{(\lambda x)^{k} t}{k^{k}}\right)
\end{aligned}
$$

where $D$ is a matrix in $\mathbb{C}^{N \times N}$ satisfying the condition $\operatorname{Re}(\mu)>-1 \forall \mu \in$ $\sigma(D)$ and all matrices are commutative.

Corollary 4.3. Let

$$
\Lambda_{\mu, \nu}(y ; z)=\sum_{r=0}^{\infty} a_{r} Z_{\mu+\nu r}^{(D, \lambda)}(y ; k) z^{r} ; a_{r} \neq 0, \mu, \nu \in \mathbb{N}_{0}
$$

and

$$
\begin{aligned}
\Psi_{n, m, \mu, \nu}(x ; y ; \eta)= & \sum_{r=0}^{\left[\frac{1}{m} n\right]} a_{r}\left[(A+I)_{k(n-m r)}\right]^{-1}(P+Q+I)_{n-m r} \\
& \times\left[(P+I)_{k(n-m r)}\right]^{-1} J_{n-m r}^{(A, B)}(x ; k) Z_{\mu+\nu r}^{(D, \lambda)}(y ; k) \eta^{r} ; n, m \in \mathbb{N},
\end{aligned}
$$

where $D$ is a matrix in $\mathbb{C}^{N \times N}$ satisfying the condition $\operatorname{Re}(\mu)>-1 \forall \mu \in$ $\sigma(D)$ and all matrices are commutative, then we have

$$
\begin{aligned}
& \sum_{n=0}^{\infty} \Psi_{n, m, \mu, \nu}\left(x ; y ; \frac{\eta}{t^{m}}\right) t^{n}=(1-t)^{-A-B-I}{ }_{k+1} F_{k}\left(\frac{A+B+I}{k+1},\right. \\
& \frac{A+B+2 I}{k+1}, \ldots, \frac{A+B+(k+1) I}{k+1} ; \frac{A+I}{k}, \frac{A+2 I}{k}, \ldots, \\
& \left.\frac{A+k I}{k} ;\left(\frac{(k+1)(1-x)}{2 k}\right)^{k}\left(\frac{-(k+1) t}{(1-t)^{k+1}}\right)\right) \Lambda_{\mu, \nu}(y ; \eta)
\end{aligned}
$$

provided that each member of (4.9) exists.

Remark 4.3. Using the generating matrix function (4.8) for the Konhauser matrix polynomials $Z_{r}^{(D, \lambda)}(y ; k)$ and taking $a_{r}=\left[(D+I)_{k r}\right]^{-1}, \mu=0$ and $\nu=1$, we have 


$$
\begin{aligned}
& \sum_{n=0}^{\infty} \sum_{r=0}^{\left[\frac{1}{m} n\right]}(A+B+I)_{n-m r}\left[(A+I)_{k(n-m r)}\right]^{-1} J_{n-m r}^{(A, B)}(x ; k) \\
& \times\left[(D+I)_{k r}\right]^{-1} Z_{r}^{(D, \lambda)}(y ; k) \eta^{r} t^{n-m r}=(1-t)^{-A-B-I} \\
& \times{ }_{k+1} F_{k}\left(\frac{A+B+I}{k+1}, \frac{A+B+2 I}{k+1}, \ldots, \frac{A+B+(k+1) I}{k+1} ;\right. \\
&\left.\frac{A+I}{k}, \frac{A+2 I}{k}, \ldots, \frac{A+k I}{k} ;\left(\frac{(k+1)(1-x)}{2 k}\right)^{k}\left(\frac{-(k+1) t}{(1-t)^{k+1}}\right)\right) \\
& \times e^{\eta}{ }_{0} F_{k}\left(-; \frac{D+I}{k}, \frac{D+2 I}{k}, \ldots, \frac{D+k I}{k} ;-\frac{(\lambda y)^{k} \eta}{k^{k}}\right), \\
& \text { for }|t|<1,|x|<1 \text { and }\left|\left(\frac{(k+1)(1-x)}{2 k}\right)^{k}\left(\frac{-(k+1) t}{(1-t)^{k+1}}\right)\right|<1 .
\end{aligned}
$$

We remark that for every suitable choice of the coefficients $a_{r}\left(r \in \mathbb{N}_{0}\right)$, if the multivariables matrix function $\Omega_{\mu}\left(y_{1}, y_{2}, \ldots, y_{s}\right), s \in \mathbb{N}$ is expressed as an appropriation product of several simpler matrix functions with commutative matrices which satisfy (1.16). The assertions of Theorem 4.1 can be applied in order to derive the certain families of more multilinear and multilateral generating matrix functions for the matrix version of the multivariable $J_{n}^{(A, B)}(x ; k)$ and $K_{n}^{(A, B)}(x ; k)$.

\section{ACKNOWLEDGEMEnTs}

The author would like to thank to the referees for their valuable comments and suggestions, which have improved the quality of the paper.

\section{REFERENCES}

[1] R. Aktaş, B. Çekim, A. Çevik, Extended Jacobi matrix polynomials, Utilitas Mathematica, Vol. 92 (2013), pp. 47-64.

[2] A. Altin, R. Aktaş, E. Erkus-Duman, On a multivariable extension for the extended Jacobi polynomials, Journal of Mathematical Analysis and Applications, Vol. 353 (2009), pp. 121-133.

[3] A. Altin, B. Çekim, Some properties associated with Hermite matrix polynomials, Utilitas Mathematica, Vol. 88 (2012), pp. 171-181.

[4] A. Altin, B. Çekim, E. Erkus-Duman, Families of generating functions for the Jacobi and related matrix polynomials, Ars Combinatoria, Vol. 117 (2014), pp. 257-273. 
[5] B. Çekim, A. Altin, R. Aktaş, Some new results for Jacobi matrix polynomials, Filomat, Vol. 27, No. 4 (2013), pp. 713-719.

[6] A. Çevik, Multivariable construction of extended Jacobi matrix polynomials, Journal of Inequalities and Special Functions, Vol. 4, No. 3 (2013), pp. 6-12.

[7] E. Defez, L. Jódar, Chebyshev matrix polynomails and second order matrix differential equations, Utilitas Mathematica, Vol. 61 (2002), pp. 107-123.

[8] E. Defez, L. Jódar, A. Law, Jacobi matrix differential equation, polynomial solutions, and their properties, Computers and Mathematics with Applications, Vol. 48 (2004), pp. 789-803.

[9] N. Dunford, J. T. Schwartz, Linear Operators, part I, General Theory, Interscience Publishers, INC. New York, 1957.

[10] E. Erkuş-Duman, B. Çekim, New generating functions for Konhauser matrix polynomials, Communications Faculty of Sciences University of Ankara Series A 1: Mathematics and Statistics, Vol. 63, No. 1 (2014), pp. 35-41.

[11] L. Jódar, J. C. Cortés, Some properties of Gamma and Beta matrix functions, Applied Mathematics Letters, Vol. 11, No. 1 (1998), pp. 89-93.

[12] L. Jódar, J. C. Cortés, On the hypergeometric matrix function, Journal of Computational and Applied Mathematics, Vol. 99 (1998), 205-217.

[13] L. Jódar, J. C. Cortés, Closed form general solution of the hypergeometric matrix differential equation, Mathematical and Computer Modelling, Vol. 32 (2000), pp. $1017-1028$.

[14] J. D. E. Konhauser, Some properties of biorthogonal polynomials, Journal of Mathematical Analysis and Applications, Vol. 11 (1965), pp. 242-260.

[15] A. Shehata, Some relations on Konhauser matrix polynomials, Miskolc Mathematical Notes, Vol. 17, No. 1 (2016), pp. 605-633.

[16] A. Shehata, Some relations on Humbert matrix polynomials, Mathematica Bohemica, Vol. 141, No. 4 (2016), pp. 407-429.

[17] A. Shehata, Some relations on the generalized Bessel matrix polynomials, Asian Journal of Mathematics and Computer Research, Vol. 17, No. 1 (2017), pp. 1-25.

[18] A. Shehata, A note on Two-variable Lommel matrix functions, Asian-European Journal of Mathematics (AEJM), Vol. 11, No.1 (2018), 1850041(14 pages).

[19] A. Shehata, Some relations on generalized Rice's matrix polynomials, An International Journal Applications and Applied Mathematics, Vol. 12, No. 1 (2017), pp. $367-391$.

[20] F. Taşdelen, B. Çekim, R. Aktaş, On a multivariable extension of Jacobi matrix polynomials, Computers and Mathematics with Applications, Vol. 61, No. 9 (2011), $2412-2423$. 
[21] S. Varma, B. Çekim, F. Taşdelen, On Konhauser matrix polynomials, Ars Combinatoria, Vol. 100 (2011), pp. 193-204.

[22] S. Varma, F. Taşdelen, Biorthogonal matrix polynomials related to Jacobi matrix polynomials, Computers and Mathematics with Applications, Vol. 62, No. 10 (2011), pp. 3663-3668.

\author{
Ayman Shehata \\ Department of Mathematics \\ FACUlty of Science \\ Assiut University \\ Assiut 71516 \\ EGYPT \\ Secondary address: \\ Department of Mathematics \\ College of Science and Arts \\ UnAIZAh, QASSIM University \\ QASSIM \\ Kingdom of Saudi Arabia \\ E-mail address: drshehata2006@yahoo.com
}

